\title{
Efecto de la hipoxia en la conducta de forrajeo de Cancer setosus (Molina, 1782) (Crustacea: Decapoda) alimentado con Mytilus chilensis (Hupé, 1854)
}

\author{
Hypoxia effect on foraging behavior of Cancer setosus (Molina, 1782) \\ (Crustacea: Decapoda) feeding on Mytilus chilensis (Hupé, 1854) \\ José A. Cisterna ${ }^{1}$, Gonzalo S. Saldías² y Cristian W. Cáceres ${ }^{1}$ \\ ${ }^{1}$ Departamento de Ecología Costera. Facultad de Ciencias. Universidad Católica de la Santísima Concepción \\ Casilla 297, Concepción, Chile \\ ${ }^{2}$ Departamento de Oceanografía, Facultad de Ciencias Naturales y Oceanográficas, Universidad de Concepción \\ Casilla 160-C, Concepción, Chile \\ gsaldias@udec.cl
}

\begin{abstract}
The variability in oxygen conditions results in different responses at marine organism's foraging behavior. In this sense, the aim of the paper was to study the foraging behavior of Cancer setosus (Molina, 1782) quantified through the size of prey selection and consumption rate under hypoxia and normal level of dissolved oxygen conditions. Significant
\end{abstract}

\section{Introducción}

El oxígeno es uno de los elementos más importante en el ciclo energético de los seres vivos, esencialmente en la respiración de los organismos aeróbicos. En ambientes terrestres el oxígeno representa el $\sim 20 \%$ de la composición atmosférica, pero en ambientes marinos la disponibilidad de este gas es mucho menor, siendo un factor limitante para las respuestas de los organismos al ambiente (Randall et al. 1997).

El agua de mar es considerada hipóxica cuando los niveles de oxígeno disuelto son menores a 2,8 $\mathrm{mg} \mathrm{O}_{2} \mathrm{~L}^{-1}$ (Díaz \& Rosenberg 1995). La exposición de los organismos a un medio hipóxico es extremadamente dinámica, espacial y temporalmente, con cambios de normoxia a hipoxia y viceversa, en el ambiente natural. Estas variaciones pueden ser episódicas (minutos/horas) o crónicas (meses/semanas) (Taylor \& Eggleston 2000), dependiendo del tipo de ambiente.

Desde un punto de vista evolutivo, existen diferencias en la tolerancia a la hipoxia entre diferentes linajes de organismos marinos, siendo los teleósteos y crustáceos los mas sensibles a las fluctuaciones de oxígeno del ambiente (Gray et al. 2002). Dentro de los crustáceos, los cangrejos son habitantes conspicuos de la zona submareal de gran parte de las costas de Chile. Estos organismos depredan principalmente bivalvos, afectando differences in prey size selection and also in the consumption rate were found. These results suggest that in hypoxic conditions C. setosus prefers size preys involving less energy expenditure. In contrast, under normal oxygen conditions the major energetic budget is probably destined to growth.

Key words: Feeding, crab, oxygen level, central Chile

significativamente la dinámica poblacional de estas especies (Brante \& Hughes 2001).

Por otra parte, se ha descrito que la relación de tamaño del cuerpo de la presa y el predador es un atributo que está relacionado directamente con el éxito del forrajeo (Scharf et al. 2000), dado que determina la tasa neta de energía disponible para suplir las demandas metabólicas del individuo (Hughes 1986). De esta manera existe un tamaño óptimo de presa que determina la máxima entrada neta de energía.

La mayoría de los depredadores no consume las presas en directa proporción con su abundancia, ya que selecciona determinadas especies y/o tamaños de los individuos (Krebs \& Davies 1997); así, las diferencias en la calidad de presas están dadas principalmente por su tamaño, componentes nutricionales y tiempo de manipulación (Carriker 1981), en condiciones favorables para oxidar la materia ingerida.

Los bordes orientales de los océanos y particularmente el sector este del Pacífico sur se caracteriza por presentar amplias zonas de mínimo oxígeno, influenciando fuertemente la distribución y diversidad de las comunidades planctónicas y bentónicas marinas (Levin et al. 2002). En estos sistemas las comunidades bacterianas y meiofaunísticas alcanzan altas abundancias, posiblemente debido a la reducción de su depredación y a la alta disponibilidad de alimento (Cook et al. 2000). 
La bahía San Vicente (zona de muestreo) presenta condiciones mínimas de oxígeno principalmente debido a la alta carga de material orgánico proveniente de la industria pesquera (Mudge \& Seguel 1999) lo que se traduce en un gran déficit de oxígeno disuelto (Rudolph et al. 2002).

En este sentido es esperable que la cantidad de oxígeno disponible afecte de alguna manera los hábitos tróficos de los organismos al limitar tanto la energía disponible para la captura del alimento como la energía disponible para su degradación y asimilación. Por consiguiente, el objetivo del trabajo es estudiar la conducta de forrajeo de C. setosus (Molina, 1782), medido a través de la preferencia del tamaño de presa y la tasa de consumo en condiciones de hipoxia y normoxia.

\section{Material y métodos}

Juveniles de Cancer setosus se recolectaron durante marea baja en la zona de muestreo (ZM) ubicada en la bahía San Vicente (36 44'S; 7309'O), Chile (Fig. 1). Los ejemplares fueron trasladados al laboratorio costero (LC) y mantenidos en acuarios con aireación constante y agua de mar recirculada directamente de la ZM. Los individuos fueron aclimatados en recipientes individuales y alimentados ad-libitum, durante 15 días, con ejemplares de Mytilus chilensis Hupé, 1854 de diferentes tallas. Previo al inicio de los experimentos, los juveniles fueron mantenidos en inanición por 5 días para estandarizar la necesidad de alimento.
De los organismos recolectados, 10 fueron mantenidos en normoxia (100\% saturación de oxígeno) y otros 10 fueron sometidos a hipoxia (30\% saturación de oxígeno); cada organismo fue sometido individualmente a cada tratamiento. El nivel de hipoxia se alcanzó adicionando nitrógeno y el nivel de normoxia se mantuvo con aireación constante.

A los juveniles de $C$. setosus se les ofreció individuos de $M$. chilensis de diferentes tallas en lapsos de $6 \mathrm{~h}$, durante 3 días, y se cuantificó su preferencia por medio del conteo de individuos consumidos. El tamaño de presas ofrecidas, según longitud de la valva (LV), fue: pequeño (41,0-52,2 mm LV), mediano (52,3-64,0 mm LV) y grande (64,1-75,0 mm LV), mientras que el peso seco (PS) de los tejidos blandos varió entre $0,31 \mathrm{~g}$ y 1,71 g.

La tasa de consumo (TC) fue determinada a partir de los resultados de preferencia alimentaria, según el tiempo transcurrido entre una alimentación y otra (24 h) y el número de presas ingeridas. Para calcular la cantidad en gramos de presas ingeridas, se midió la LV de las presas y el valor fue ingresado en la ecuación de la regresión entre la LV y el PS de los tejidos blandos de M. chilensis (Fig. 2a).

Para calcular la ingesta energética de $C$. setosus se utilizó como contenido energético de $M$. chilensis el valor reportado por Duarte et al. (1980), el cual es de 712 cal $\mathrm{g}^{-1}$ peso fresco, luego este valor fue multiplicado por 4,1868 para calcular la energía en J.

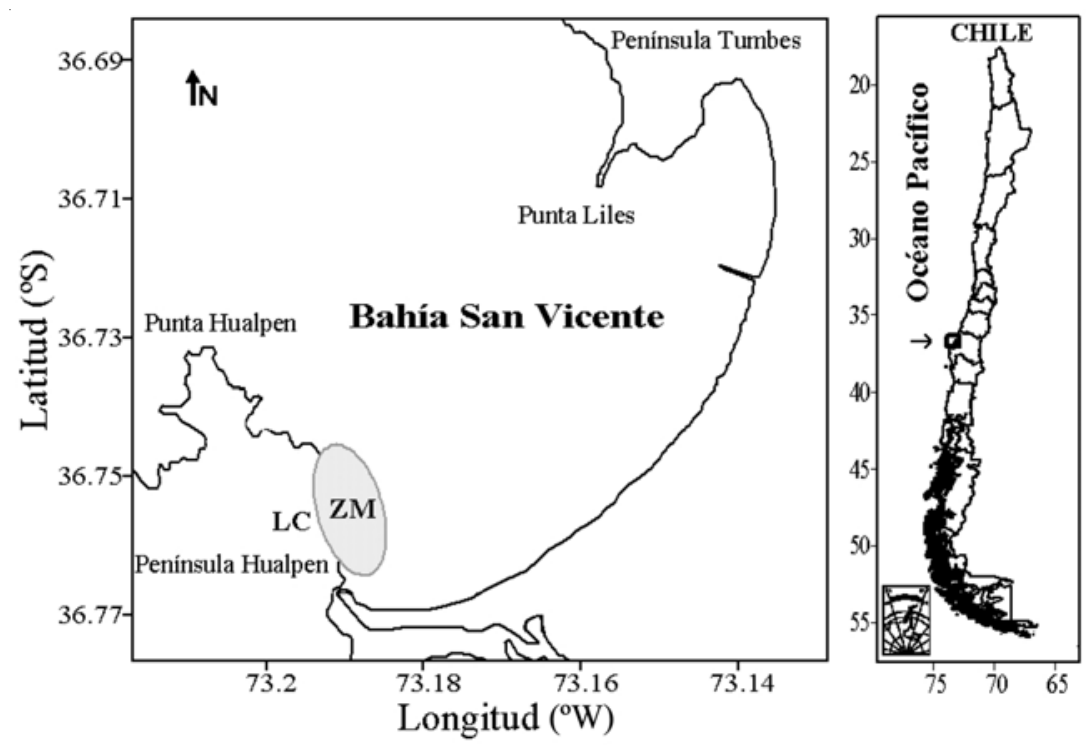

Figura 1

Zona de muestreo (ZM) y laboratorio costero (LC) ubicados en Bahía San Vicente Sampling zone (ZM) and coastal laboratory (LC) located in San Vicente Bay 

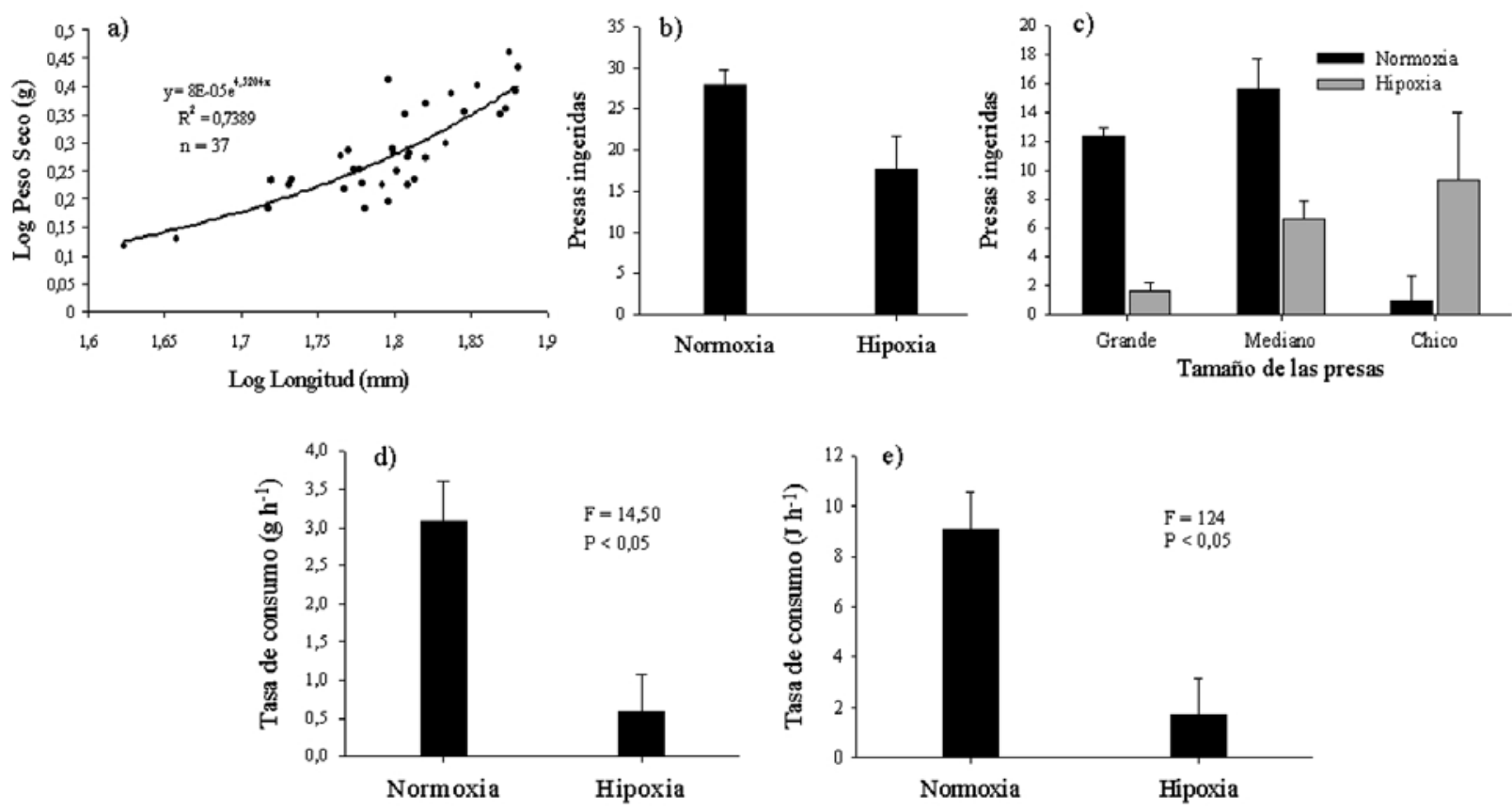

Figura 2

Conducta de forrajeo de $C$. setosus en condiciones de hipoxia y normoxia (promedio \pm desviación estándar). a) relación entre la longitud de la valva y el peso seco de los tejidos blandos de M. chilensis, b) número de presas ingeridas, c) número de presas ingeridas según el tamaño de las presas, d) tasa de consumo $\left(\mathrm{g} \mathrm{h}^{-1}\right)$, y e) tasa de consumo energético $\left(\mathrm{J} \mathrm{h}^{-1}\right)$

Foraging behavior of $C$. setosus under hypoxic and normoxic conditions (mean \pm standard deviation). a) relationship between valve length and soft tissues dry weight of $M$. chilensis, b) number of consumed preys, c) number of consumed preys according to the prey sizes, d) consumption rate $\left(\mathrm{g} \mathrm{h}^{-1}\right)$, and e) energetic consumption rate $\left(\mathrm{J} \mathrm{h}^{-1}\right)$.

Para evaluar cómo se relaciona la LV y el PS de las partes blandas de las presas, se disectaron individuos de M. chilensis de distintos tamaños de LV. A estos individuos se les separaron las valvas de las partes blandas, las que posteriormente fueron mantenidas en estufa $\left(\sim 50^{\circ} \mathrm{C}\right)$ hasta alcanzar un peso constante. Con los valores obtenidos se ajustó una relación entre la LV (mm) y el PS de la carne (g). Posteriormente, mediante la aplicación de logaritmo a los datos se determinó el coeficiente de regresión y la probabilidad asociada a la curva (Fig. 2a).

Se realizó un análisis de varianza de dos vías para evaluar las posibles diferencias en el número de presas ingeridas según el tamaño de la presa y la condición de oxígeno. Además, se efectuaron análisis de varianza para determinar posibles diferencias tanto para la tasa de consumo como para la tasa de ingesta energética (Zar 1984).

\section{Resultados}

El total de presas consumidas por los individuos de Cancer setosus en los estados de normoxia e hipoxia mostró diferencias, con $28 \pm 2$ y $18 \pm 4$ presas ingeridas respectivamente en promedio durante los tres días de experimentación (Fig. 2b). Además, presentaron diferencias en la preferencia de presa ingerida en cada condición, ya que en normoxia eligieron presas de tamaño mediano, luego tamaño grande y finalmente tamaño pequeño. Las diferencias estadísticas se presentaron entre el tamaño mediano y grande en comparación con el tamaño pequeño. Por otra parte, los organismos sometidos a hipoxia prefirieron, en primer lugar, presas de tamaño pequeño, luego mediano, y finalmente presas de tamaño grande. Las diferencias estadísticas se encontraron entre las presas de tamaño grande y pequeño, y éstas fueron iguales con relación al tamaño mediano (Fig. 2c, Tabla 1).

Se presentaron diferencias significativas en la tasa de consumo entre el ritmo de alimentación de los organismos mantenidos en normoxia con respecto a los de hipoxia (promedio de 3,084 $\pm 0,51 \mathrm{~g} \mathrm{~h}^{-1}$ y 0,58 $\pm 0,48 \mathrm{~g} \mathrm{~h}^{-1}$ respectivamente) (Fig. 2d). Además se encontraron diferencias significativas en la cantidad de energía ingerida en ambas condiciones, con un ritmo de 
Tabla 1

\section{Análisis de varianza de dos vías para el consumo de presas por $C$. setosus según el tamaño de las presas y el nivel de oxígeno}

Two-way analysis of variance for the prey consumptions by C. setosus according to prey size and oxygen level

\begin{tabular}{lrrcc}
\hline Origen & g.l. & \multicolumn{1}{c}{ CM } & F & $P$ \\
\hline Tamaño & 2 & 56,72 & 11,09 & 0,001 \\
Oxígeno & 1 & 64,22 & 12,56 & 0,004 \\
Interacción & 2 & 166,05 & 32,48 & 0,001 \\
Error & 12 & 5,11 & & \\
\hline
\end{tabular}

alimentación de 9,19 \pm 1,54 $\mathrm{J} \mathrm{h}^{-1}$ en normoxia y 1,75 \pm 1,43 $\mathrm{J} \mathrm{h}^{-1}$ en hipoxia (Fig. 2e).

\section{Discusión}

La preferencia de los individuos de Cancer setosus mantenidos en normoxia por presas de tamaño mediano indica que ese tamaño corporal de presa es el que entrega mayor retorno energético; no así los tamaños pequeños, los cuales presentan una concha más blanda, pero la cantidad de tejido blando es muy reducida. Por otra parte, las presas de tamaños mayores presentan una concha más dura, por lo que romperla para conseguir el alimento necesita de una mayor energía, y no constituye el primer ítem en la preferencia de tallas. De la misma forma que para crustáceos, se ha descrito que gasterópodos murícidos tienen una preferencia alimentaria por presas que presentan una concha que es más fácil de romper en comparación con las de concha más dura (Hughes 1986), por lo que la maximización de la energía es una variable que determina la respuesta de los organismos. Esto explica, además, la preferencia de $C$. setosus por presas pequeñas en estado de hipoxia.

Una vez que el animal ha realizado la categorización de las presas, dentro de la teoría de forrajeo, específicamente, el modelo de selección de presas, predice que aquellas de mayor calidad energética serán seleccionadas en primer lugar, mientras que la ingestión de las presas energéticamente subóptimas dependerá de la disponibilidad de presas óptimas en el ambiente (Burrows \& Hughes 1991).

Por otro lado, se ha descrito que un déficit en la disponibilidad de oxígeno puede afectar incluso la relación predador-presa al cambiar el orden de preferencia de los ítems tróficos (Sandberg 1994). El efecto negativo de la hipoxia en la conducta alimenticia se presenta por no poder incrementar el metabolismo aeróbico más allá de la tasa basal (Brante \& Hughes 2001). El metabolismo anaeróbico, en comparación con el metabolismo aeróbico, es muy ineficiente en la producción de energía, por lo que los organismos sometidos a hipoxia, quienes probablemente tuvieron que recurrir a metabolismo anaeróbico, deben ocupar su energía en mantenimiento y no en crecimiento (Shin et al. 2005).

Por lo tanto, C. setosus elige el tamaño de presa, lo que sugiere que el tamaño de presa elegido en cada condición es el tamaño que entrega el mayor retorno energético en relación a la energía invertida en su consumo.

Además, C. setosus destina la energía según el estado en el cual se encuentre. Esto se evidencia por la diferencia encontrada en la tasa de alimentación. El ritmo de alimentación menor presentado por los organismos sometidos a hipoxia revela que estos organismos destinan su energía a mantenerse y no a crecimiento como sí puede sugerirse para los organismos mantenidos en normoxia.

\section{Agradecimientos}

Se agradecen los atinados comentarios y sugerencias de los revisores anónimos y editores de la revista para mejorar la estructura del trabajo.

\section{Literatura citada}

Brante A \& RN Hughes. 2001. Effects of hypoxia on the prey-handling behaviour of Carcinus maenas feeding on Mytilus edulis. Marine Ecology Progress Series 209: 301305.

Burrows MT \& RN Hughes. 1991. Optimal foraging decisions by dogwhelks, Nucella lapillus (L.): influences of mortality risk and rate-constrained digestion. Functional Ecology 5: 461-475.

Carriker MR. 1981. Shell penetration and feeding by naticacean and muricacean predatory gastropods: a synthesis. Malacologia 20: 403-422.

Cook AA, PJ Lambshead, LE Hawkins, N Mitchell \& L Levin. 2000. Nematode abundance at the oxygen minimum zone in the Arabian Sea. Deep-Sea Research 47: 75-85.

Diaz RJ \& R Rosenberg. 1995. Marine benthic hypoxia: a review of its ecological effects and behavioural responses of benthic macrofauna. Oceanography and Marine Biology Annual Review 33: 245-303.

Duarte W, F Jara \& C Moreno. 1980. Contenido energético de algunos invertebrados bentónicos de la costa de Chile y fluctuación anual en Mytilus chilensis Hupé 1854. Boletim do Instituto Oceanográfico de São Paulo 29: 157162. 
Gray J, RSS Wu \& YY Or. 2002. Effects of hypoxia and organic enrichment on the coastal marine environment. Marine Ecology Progress Series 238: 249-279.

Hughes RN. 1986. A functional biology of marine gastropods, 245 pp. The Johns Hopkins University Press, Baltimore.

Krebs JR \& NB Davies. 1997. Behavioural ecology: an evolutionary approach. 456 pp. Blackwell Science, Oxford.

Levin L, D Gutiérrez, A Rathburn, C Neira, J Sellanes, P Muñoz, V Gallardo \& M Salamanca. 2002. Benthic processes on the Peru margin: A transect across the oxygen minimum zone during the 1997-1998 El Niño. Progress in Oceanography 53: 1-27.

Mudge S \& CG Seguel. 1999. Organic contamination of San Vicente Bay, Chile. Marine Pollution Bulletin 38: 10111021.

Randall D, W Burggren \& K French. 1997. Animal Physiology: mechanisms and adaptation, 727 pp. WH Freeman \& Company, Nueva York.

Rudolph A, R Ahumada \& C Pérez. 2002. Dissolved oxygen content as an index of water quality in San Vicente Bay, Chile (36 $45^{\prime}$ 'S). Environmental Monitoring and Assessment 78: 89-100.
Sandberg E. 1994. Does short-term oxygen depletion affect predator-prey relationships in zoobenthos? Experiments with the isopod Saduria entomon. Marine Ecology Progress Series 103: 73-80.

Scharf F, F Juanes \& R Rountree. 2000. Predator size-prey size relationships of marine fish predators: interspecific variation and effects of ontogeny and body size on trophicniche breadth. Marine Ecology Progress Series 208: 229248.

Shin P, P Cheung, F Yang \& S Cheung. 2005. Intermittent exposure to reduced oxygen levels affects prey-size selection and consumption in swimming crab Thalamita danae Stimpson. Marine Pollution Bulletin 51: 10031009.

Taylor DL \& DB Eggleston. 2000. Effects of hypoxia on an estuarine predator-prey interaction: foraging behavior and mutual interference in the blue crab Callinectes sapidus and the infaunal clam prey Mya arenaria. Marine Ecology Progress Series 196: 221-237.

Zar J. 1984. Biostatistical Analysis, 718 pp. Prentice-Hall, New Jersey.

Recibido el 10 de marzo de 2008 y aceptado el 26 de mayo de 2008 